\title{
Platelets Regulate the Migration of Keratinocytes via Podoplanin/CLEC-2 Signaling during Cutaneous Wound Healing in Mice
}

Jun Asai, ${ }^{*}$ Satoshi Hirakawa, ${ }^{\dagger}$ Jun-ichi Sakabe, ${ }^{\dagger}$ Tsunao Kishida, ${ }^{\dagger}$ Makoto Wada, ${ }^{*}$ Naomi Nakamura, ${ }^{*}$ Hideya Takenaka, ${ }^{*}$ Osam Mazda, ${ }^{\ddagger}$ Tetsumei Urano, ${ }^{\S}$ Katsue Suzuki-Inoue, ${ }^{\llbracket}$ Yoshiki Tokura, ${ }^{\dagger}$ and Norito Katoh*

\author{
From the Departments of Dermatology* and Immunology, ${ }^{\ddagger}$ Kyoto Prefectural University of Medicine, Kyoto; the Departments of Dermatology ${ }^{\dagger}$ and \\ Physiology, ${ }^{\S}$ Hamamatsu University School of Medicine, Hamamatsu; and the Department of Clinical and Laboratory Medicine, "Faculty of Medicine, \\ University of Yamanashi, Kofu, Japan
}

\author{
Accepted for publication \\ September 24, 2015. \\ Address correspondence to \\ Jun Asai, M.D., Ph.D., \\ Department of Dermatology, \\ Kyoto Prefectural University \\ of Medicine, 465 Hirokoji, \\ Kawaramachi, Kamigyo-Ku, \\ Kyoto 602-8566, Japan. \\ E-mail: jasai@koto.kpu-m.ac.jp.
}

\begin{abstract}
Podoplanin is an endogenous ligand for C-type lectin-like receptor 2 (CLEC-2), which is expressed on platelets. Recent evidence indicates that this specific marker of lymphatic endothelial cells is also expressed by keratinocytes at the edge of wounds. However, whether podoplanin or platelets play a role in keratinocyte activity during wound healing remains unknown. We evaluated the effect of podoplanin expression levels on keratinocyte motility using cultured primary normal human epidermal keratinocytes (NHEKs). Down-regulation of podoplanin in NHEKs via transfection with podoplanin siRNA inhibited their migration, indicating that podoplanin plays a mandatory role in this process. In addition, downregulation of podoplanin was correlated with up-regulation of E-cadherin, suggesting that podoplaninmediated stimulation of keratinocyte migration is associated with a loss of E-cadherin. Both the addition of platelets and treatment with CLEC-2 inhibited the migration of NHEKs. The down-regulation of RhoA activity and the up-regulation of E-cadherin in keratinocytes were also induced by CLEC-2. In conclusion, these results suggest that podoplanin/CLEC-2 signaling regulates keratinocyte migration via modulating E-cadherin expression through RhoA signaling. Altering the regulation of keratinocyte migration by podoplanin might be a novel therapeutic approach to improve wound healing. (Am J Pathol 2016, 186: 101-108; http://dx.doi.org/10.1016/j.ajpath.2015.09.007)
\end{abstract}

Podoplanin, a small 38-kDa mucin-type transmembrane glycoprotein, is expressed in kidney podocytes (the origin of its nomenclature), type I lung alveolar epithelial cells, and lymphatic endothelial cells. ${ }^{1,2}$ Recently, podoplanin has been implicated in tumor progression. ${ }^{3-6}$ Podoplanin expression is up-regulated in the invasive front of several human carcinomas, including squamous cell carcinomas of the oral cavity, lung, and skin. ${ }^{7,8}$ The expression of this glycoprotein is correlated with the down-regulation of the cell-cell adhesion protein E-cadherin, ${ }^{9}$ which leads to tumor invasion via stimulation of the epithelial-mesenchymal transition. In addition to tumor cells, epidermal keratinocytes express podoplanin under several pathological conditions, such as wound healing and psoriasis. ${ }^{10,11}$ However, the role of podoplanin in normal epidermal keratinocytes during wound healing remains unknown.
C-type lectin-like receptor 2 (CLEC-2) is a receptor for the platelet-activating snake venom, rhodocytin. ${ }^{12}$ Podoplanin was identified as an endogenous ligand for CLEC-2 ${ }^{13}$ and induces platelet aggregation by binding to CLEC-2. This contributes to thrombosis/hemostasis, lymphangiogenesis, and tumor metastasis. ${ }^{13,14}$ During wound healing processes, platelet aggregation and clot formation are the initial steps of tissue regeneration. Platelet-rich aggregates release various growth factors and cytokines to promote wound healing. Platelet lysates stimulate the proliferation and migration of immortalized HaCaT keratinocytes, ${ }^{15}$ although this cell line does not express podoplanin. ${ }^{16}$ However, the physiological significance of

Supported by JSPS KAKENHI grant 25461674 (J.A.).

Disclosures: None declared. 
the podoplanin/CLEC-2 interaction between platelets and normal keratinocytes remains to be investigated.

Herein, we investigated the function of podoplanin in normal keratinocytes, with special focus on the alterations in motility induced by podoplanin siRNA and the interaction between platelets and keratinocytes. The results suggest that podoplanin plays a mandatory role in keratinocyte migration and that platelets inhibit the migration of keratinocytes. We further determined that the regulatory effects of platelets on keratinocytes were mediated by podoplanin/ CLEC-2 signaling.

\section{Materials and Methods}

\section{Wound Induction}

Male C57BL/6 mice used in this study were between 8 and 10 weeks of age at the time of the study. Wounds were induced as described previously. ${ }^{17-20}$ In brief, after induction of deep anesthesia by i.p. injection of $160 \mathrm{mg} / \mathrm{kg}$ sodium pentobarbital, full-thickness excisional skin wounds were made on the backs of mice using 8-mm skin biopsy punches, with one wound generated in each mouse. Each wound was covered with semipermeable polyurethane dressing (OpSite; Smith and Nephew, Massillon, $\mathrm{OH}$ ). Wound tissues were harvested on days 1, 3, 7, and 10 after wound induction. All animal protocols were approved by the Kyoto Prefectural University of Medicine (Kyoto, Japan) Institutional Animal Care and Use Committee, and were consistent with the Guide for the Care and Use of Laboratory Animals. ${ }^{21}$

\section{Cell Culture}

Primary normal human epidermal keratinocytes (NHEKs; Kurabo, Osaka, Japan) were cultured in HuMedia-KG2 serum-free medium (Kurabo).

\section{Platelet Preparation}

Venous blood from a healthy drug-free patient, who was scheduled to have plastic surgery, was collected into $10 \%$ sodium citrate at the time of their blood test. This study was approved by the Ethical Committee of the Kyoto Prefectural University of Medicine, and written informed consent was provided according to the Declaration of Helsinki. Platelets were obtained by centrifugation, as described previously, using prostacyclin to prevent activation during the isolation procedure. $^{22}$

\section{siRNA Transfection}

Control or podoplanin siRNA (50 nmol/L; Hs[lowen]PDPN [lowen]1 FlexiTube siRNA, catalog number SI03060260; Qiagen, Düsseldorf, Germany) was transfected into NHEKs using Lipofectamine RNAiMAX (Invitrogen, Carlsbad, $\mathrm{CA}$ ), according to the manufacturer's protocol. For RT-PCR
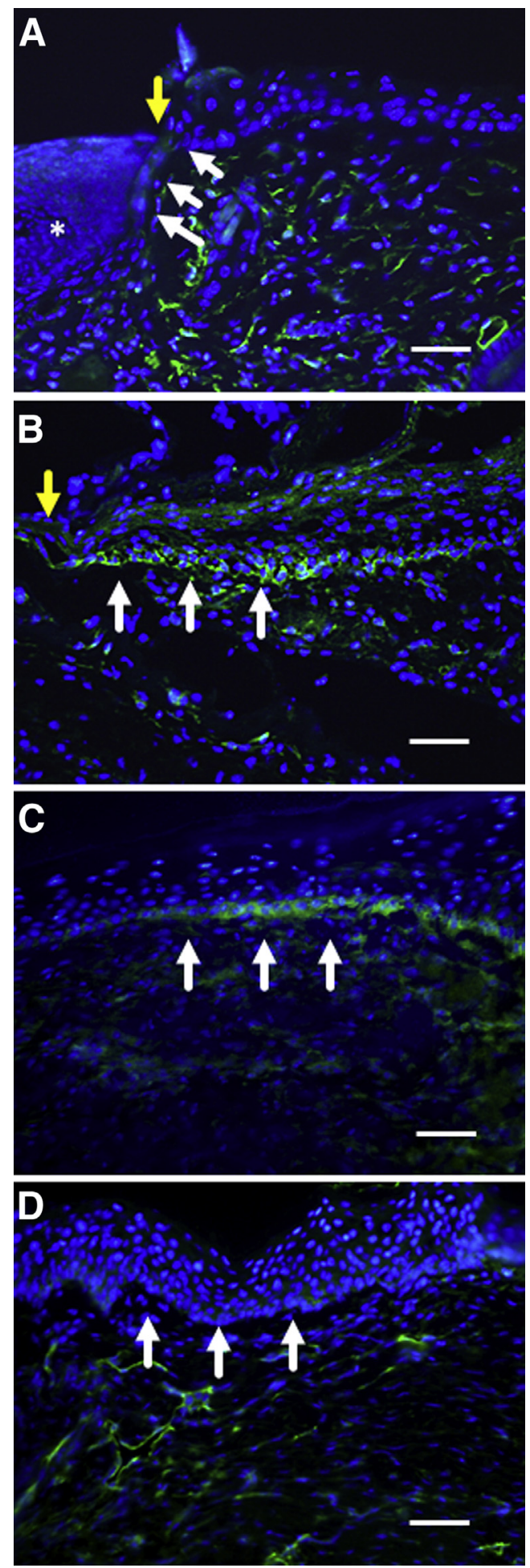

Figure 1 Time-dependent expression of podoplanin in keratinocytes during wound healing. Green and blue fluorescence corresponds to podoplanin-positive cells and DAPI-labeled nuclei, respectively. Wound edge during the inflammatory phase (day $1 ; \mathbf{A}$ ), during the tissue formation phase (day $3 ; \mathbf{B}$ ), at the period of transition from the tissue formation phase to the tissue remodeling phase (day $7 ; \mathbf{C}$ ), and at the end of the tissue remodeling phase (day $10 ; \mathbf{D}$ ). The asterisk indicates platelet aggregates covering the surface of the wound; yellow arrows, the leading edge of the epidermis; white arrows, the basal cell layer of the epidermis at the wound edge. Scale bars: $50 \mu \mathrm{m}$ (A-D). Original magnification, $\times 100$ (A-D). 


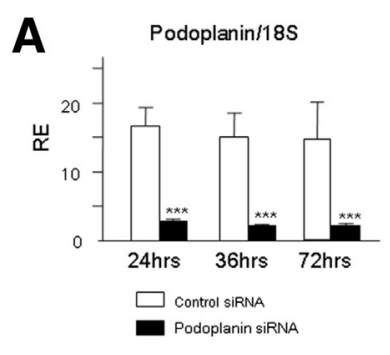

D

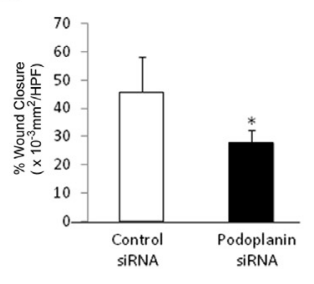

B

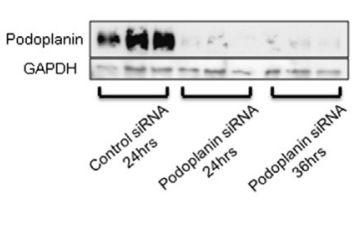

E

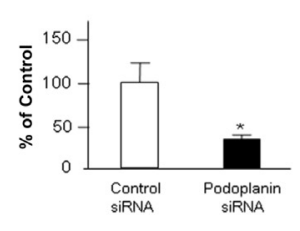

C

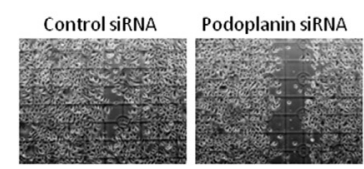

Figure 2 Efficacy of podoplanin siRNA in normal human epidermal keratinocytes (NHEKs). NHEKs were harvested 24, 36, and 72 hours after transfection. A: Quantitative RT-PCR of podoplanin mRNA in NHEKs treated with control or podoplanin siRNA. B: Western blot analysis of podoplanin protein expression in NHEKs treated with control or podoplanin siRNA. C: The effects of podoplanin down-regulation on NHEK migration. The migration of NHEKs transfected with control or podoplanin siRNA was investigated by the scratch wound assay. D: Graphical presentation of the wound closure areas, quantified as described in Materials and Methods. E: RhoA activity of NHEKs measured by the Great Lakes Integrated Sciences + Assessments Center assay. Control or podoplanin siRNA transfected cells were harvested at 24 hours after transfection. ${ }^{*} P<0.05,{ }^{* * *} P<0.001$ versus control siRNA. $n=6$ in each group (A and $\mathbf{D}$ ); $n=3$ in each group (B and $\mathbf{E}$ ). GAPDH, glyceraldehyde-3phosphate dehydrogenase; HPF, high-power field; $R E$, relative expression.

and the scratch wound assay, cells were used 24 hours after transfection.

\section{Immunofluorescence Staining}

Sections of wounds were stained with a hamster anti-mouse podoplanin antibody (Angiobio, Del Mar, CA). Podoplanin labeling was visualized with a fluorescein-conjugated antihamster antibody (Vector Laboratories, Burlingame, CA). E-cadherin and $\mathrm{N}$-cadherin expression was evaluated using goat anti-mouse E-cadherin and $\mathrm{N}$-cadherin antibodies (R\&D Systems, Minneapolis, MN), respectively, and a Cy3conjugated anti-goat antibody (Vector Laboratories). Platelets were evaluated using a rat monoclonal anti-mouse CD41 antibody (AbD Serotec, Oxford, UK) labeled with a Cy3-conjugated anti-rat antibody (Vector Laboratories). NHEKs treated with $100 \mathrm{ng} / \mathrm{mL}$ CLEC-2 or vehicle for 72 hours were stained with a mouse polyclonal anti-human E-cadherin (R\&D Systems), rabbit monoclonal $\beta$-catenin (Abcam, Cambridge, UK), or rabbit polyclonal N-cadherin (R\&D Systems) antibody labeled with fluorescein isothiocyanate-conjugated anti-mouse or anti-rabbit antibodies (Vector Laboratories), as appropriate.

\section{RNA Isolation, cDNA Synthesis, and Quantitative Real-Time PCR}

RNA was extracted using ISOGENII (Nippon Gene, Toyama, Japan), according to the manufacturer's instructions, and processed for cDNA synthesis and quantitative RT-PCR, as previously described. ${ }^{19}$ To evaluate the efficacy of podoplanin siRNA, NHEKs were harvested 24 and 36 hours after transfection. To evaluate the effects of platelets on NHEKs, NHEKs were harvested 24 hours after the addition of $2.0 \times 10^{5}$ platelets $/ \mathrm{mL}$. Recombinant human CLEC-2 (hCLEC-2; R\&D Systems) was added to cultured
NHEKs at the indicated concentrations, and cells were harvested after 24 hours. Podoplanin, E-cadherin, and $\mathrm{N}$-cadherin mRNA levels were normalized on the basis of the level of 18S, an internal reference gene. The primers used in the study were obtained from Qiagen (podoplanin, catalog number QT01015084; E-cadherin, catalog number QT00080143; N-cadherin, catalog number QT00063196; 18S, catalog number QT00199367).

\section{SDS-PAGE and Western Blot Analysis}

Cells were lysed with radioimmunoprecipitation assay buffer (Invitrogen) and sonicated. After sonication, cell lysates were centrifuged at $13,000 \times g$ for 20 minutes at $4^{\circ} \mathrm{C}$, and the supernatants were collected into fresh tubes. We then added $4 \times$ SDS sample buffer with $0.1 \mathrm{~mol} / \mathrm{L}$ dithiothreitol to each sample. Samples were heated for 5 minutes at $95^{\circ} \mathrm{C}$, and 20 $\mu \mathrm{g}$ protein was separated by $10 \%$ SDS-PAGE and electroblotted onto polyvinylidene difluoride membranes for 2 hours at $180 \mathrm{~mA}$. The membranes were incubated with a rabbit anti-human podoplanin polyclonal antibody (R\&D Systems) or a mouse anti-glyceraldehyde-3-phosphate dehydrogenase monoclonal antibody (Santa Cruz Biotechnology, Dallas, TX) and detected with horseradish peroxidase-conjugated goat anti-rabbit $\mathrm{IgG}$ (Bio-Rad, Hercules, CA) or horseradish peroxidase-conjugated goat anti-mouse IgG (Bio-Rad). Immunoblots were visualized using an ECL Plus Western Blotting Detection Reagents Kit (GE Healthcare, Little Chalfont, Buckinghamshire, UK), according to the manufacturer's protocol.

\section{Scratch Wound Assay}

Scratch wounds were generated in confluent monolayers of NHEKs by using a 1-mL pipette tip. To evaluate the effects of podoplanin siRNA, NHEKs were used 24 hours after the 

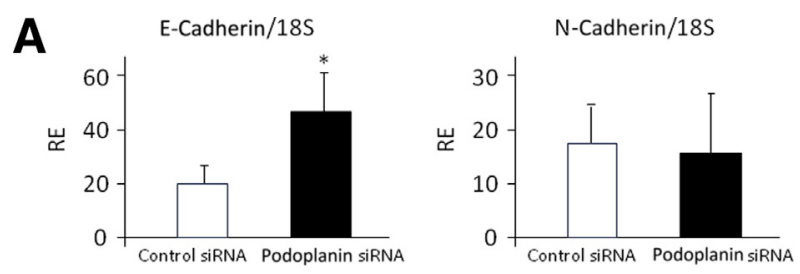

B
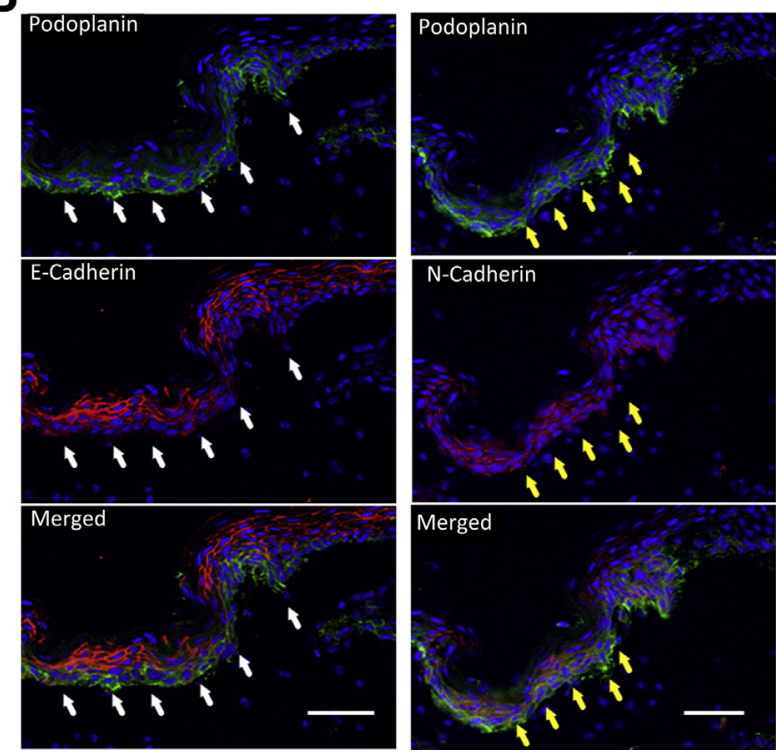

Figure 3 Cadherin expression in normal human epidermal keratinocytes (NHEKs) and keratinocytes at the wound edge. A: Quantitative RT-PCR of $\mathrm{E}$-cadherin and $\mathrm{N}$-cadherin mRNA in NHEKs transfected with control or podoplanin siRNA. B: Representative images of the immunostained wound edge at 3 days after wound generation. Red and green correspond to E-cadherin (left panels) or N-cadherin (right panels) and podoplanin, respectively. Blue fluorescence indicates DAPI-labeled nuclei. The yellow arrows indicate the leading edge of the epidermis; white arrows, basal cell layer of the epidermis at the wound edge. ${ }^{*} P<0.05$ versus control siRNA. $n=6$ in each group (A). Scale bar $=50 \mu \mathrm{m}$ (B). Original magnification, $\times 200$ (B). RE, relative expression.

transfection. After washing away suspended cells, cultures were refed with medium. To evaluate the effects of platelets or CLEC- 2 on NHEKs, $2.0 \times 10^{5}$ platelets $/ \mathrm{mL}$, or 10,100 , or $250 \mathrm{ng} / \mathrm{mL}$ hCLEC-2 was added to the NHEKs, respectively. Cell migration into the wound space was estimated at 18 hours after wounding. Images were analyzed using ImageJ software version $1.47 \mathrm{v}$ (NIH, Bethesda, MD; http://imagej.nih.gov/ij) ${ }^{23}$ by tracing the wound margin with a high-resolution computer mouse and calculating the total pixel area.

\section{Measurement of RhoA Activity}

The direct activation of RhoA was evaluated using ELISA based small G-protein activation (G-LISA) assay (Cytoskeleton Inc., Denver, CO), according to the manufacturer's protocol. Briefly, NHEKs at $30 \%$ to $50 \%$ confluence, seeded onto 12-well flat-bottomed plates, were treated with 100 $\mathrm{ng} / \mathrm{mL}$ CLEC-2. After incubation for $1,5,10$, or 30 minutes at $37^{\circ} \mathrm{C}$, cells were treated with lysis buffer. Rho-GTP in the cell lysate was detected using a RhoA G-LISA kit. The absorbance at $490 \mathrm{~nm}$ was recorded using a 96-well enzyme-linked immunosorbent assay plate reader.

\section{Statistical Analysis}

All results are presented as the means \pm SEM. Statistical comparisons between two groups were performed by $t$-test. Multiple groups were analyzed by one-way analysis of variance, followed by appropriate post hoc tests to determine statistical significance. $P<0.05$ was considered significant. All in vitro experiments were performed at least in triplicate.

\section{Results}

\section{Expression of Podoplanin by Keratinocytes during Wound Healing}

To evaluate the time-dependent expression of podoplanin during wound healing, full-thickness wounds were generated on the dorsal skin of mice (Figure 1). Podoplanin expression started to be observed at day 1 , which represented the inflammatory phase in the wound healing process. Podoplanin expression was highly up-regulated at days 3 and 7 after
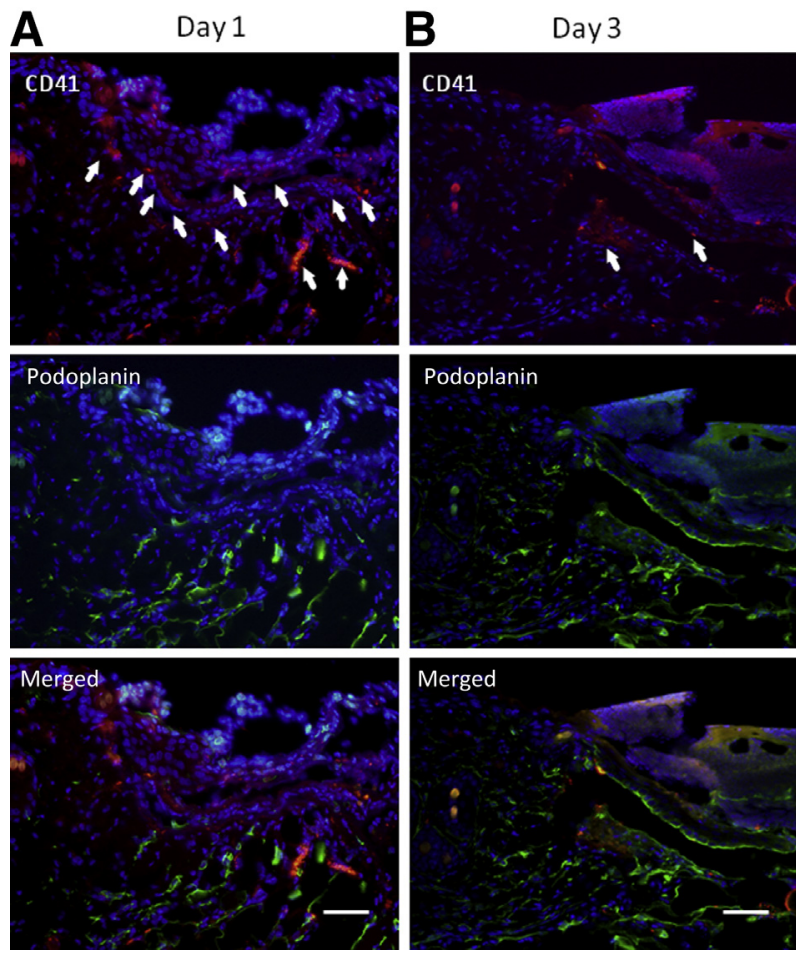

Figure 4 Direct interaction of platelets and keratinocytes during wound healing. Green, red, and blue fluorescence corresponds to podoplaninpositive cells, CD41-positive cells (platelets), and DAPI-labeled nuclei, respectively. Wound edge during the inflammatory phase (day $1 ; \mathbf{A})$ and during the tissue formation phase (day 3; B). White arrows indicate the accumulation of platelets on the basal cell layer of the epidermis at the wound edge. Scale bar $=50 \mu \mathrm{m}(\mathbf{A}$ and $\mathbf{B})$. Original magnification, $\times 100$ (A and B). 

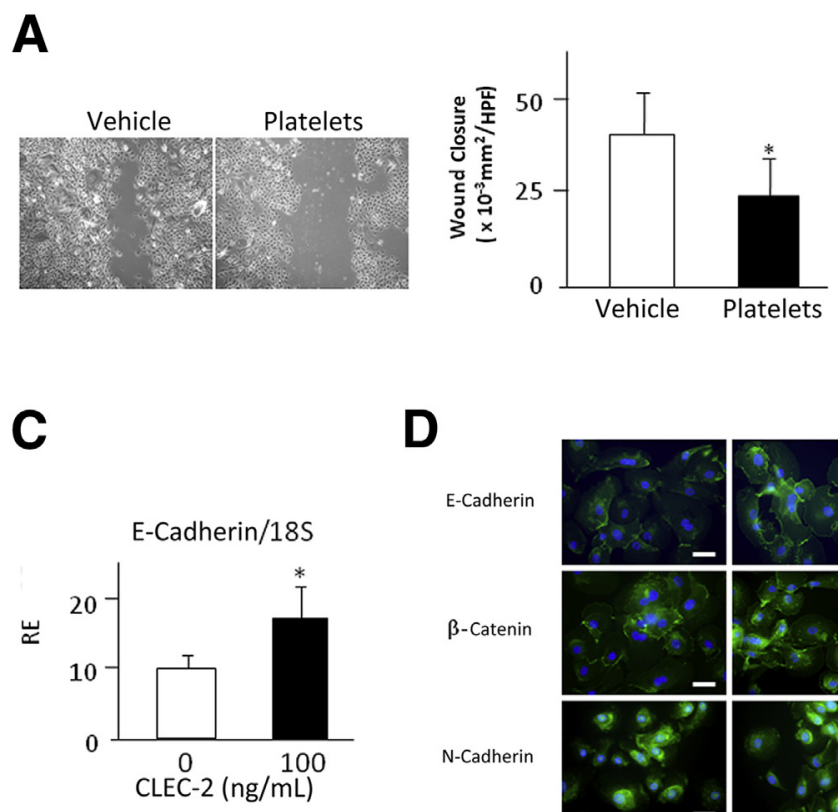

D
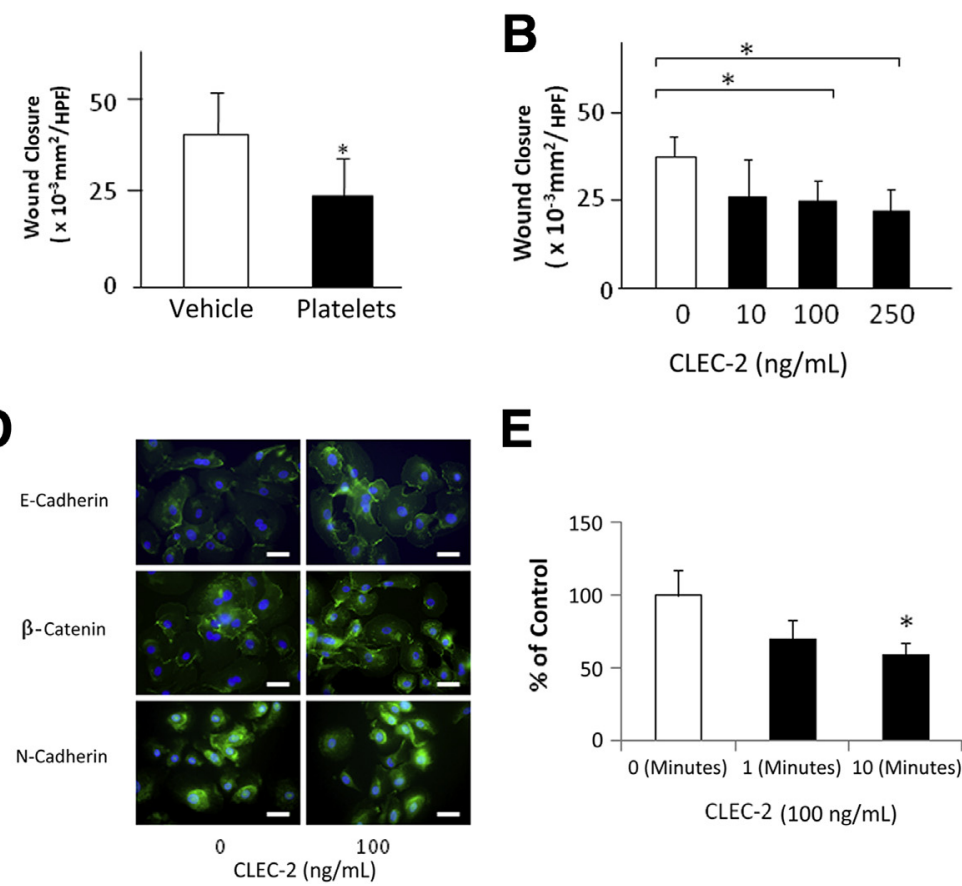

$\mathbf{E}$

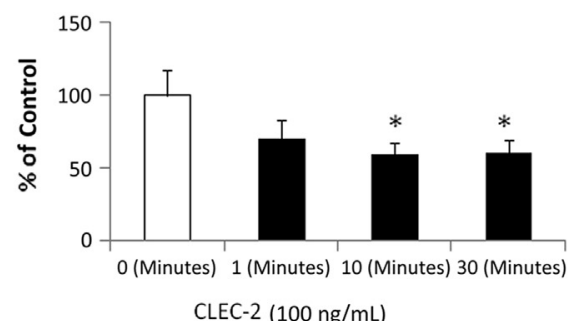

CLEC-2 $(100 \mathrm{ng} / \mathrm{mL})$

Figure 5 Effects of platelets on keratinocyte motility. A: Migration of normal human epidermal keratinocytes (NHEKs) treated with either $2.0 \times 10^{5}$ platelets $/ \mathrm{mL}$ or vehicle was investigated by the scratch wound assay. Graphical presentation of the wound closure area, quantified as described in Materials and Methods. B: Graphical representation of the wound closure area after treatment with 10, 100, or $250 \mathrm{ng} / \mathrm{mL}$ human C-type lectin-like receptor 2 (hCLEC), or with vehicle, quantified as described in Materials and Methods. C: Quantitative RT-PCR of E-cadherin mRNA in NHEKs treated with $100 \mathrm{ng} / \mathrm{mL} \mathrm{hCLEC}-2$ or vehicle. D: Immunofluorescence staining of E-cadherin, $\beta$-catenin, and N-cadherin of NHEKs treated with $100 \mathrm{ng} / \mathrm{mL}$ CLEC-2. E: RhoA activity of NHEKs measured by the Great Lakes Integrated Sciences + Assessments Center assay. To each culture, $100 \mathrm{ng} / \mathrm{mL} \mathrm{hCLEC-2} \mathrm{was} \mathrm{added,} \mathrm{and} \mathrm{cells} \mathrm{were} \mathrm{harvested} \mathrm{at} \mathrm{the}$ indicated time point. ${ }^{*} P<0.05$ versus control siRNA. $n=6$ in each group $(\mathbf{A}-\mathbf{C}) ; n=3$ in each group (E). Scale bar $=50 \mu \mathrm{m}(\mathbf{D})$. HPF, high-power field; $\mathrm{RE}$, relative expression.

wounding, and it had decreased by day 10 when the wound was completely closed.

\section{Effects of Podoplanin siRNA on NHEKs}

To examine the efficacy of podoplanin siRNA, podoplanin mRNA and protein levels were analyzed by quantitative RT-PCR and Western blot analysis, respectively. Transfection of podoplanin siRNA into NHEKs resulted in significant decrements in both the mRNA (Figure 2A) and protein (Figure 2B) levels up to 72 and 36 hours after transfection, respectively, compared with those after transfection of the control siRNA $(P<0.001)$.

We next investigated the effects of down-regulating podoplanin on the motility of NHEKs using the scratch wound assay (Figure 2C). Twenty-four hours after transfection with podoplanin siRNA, a scratch wound was generated on a confluent monolayer of NHEKs and the noncell area was measured. Eighteen hours after the scratch, the non-cell area was again measured. The wound closure area was analyzed by subtracting the latter from the former area. Podoplanin siRNA transfection significantly inhibited the migration of NHEKs by $40 \%$ (Figure 2D). To confirm that these results were induced specifically by podoplanin down-regulation, we performed the experiments using a different podoplanin siRNA (Hs[lowen]T1A-2[lowen]7, catalog S102625049; Qiagen) and obtained similar results (Supplemental Figure S1).

To evaluate the mechanisms of podoplanin-mediated cell motility, RhoA activity, which is one of the key mediators of cell migration, was directly measured by G-LISA. RhoA activity was down-regulated by podoplanin siRNA knockdown (Figure 2E), suggesting that podoplanin mediates keratinocyte motility partly via the RhoA signaling pathway.

Because the down-regulation of E-cadherin and the upregulation of $\mathrm{N}$-cadherin promote cell motility and the epithelial-mesenchymal transition in several tumor cells, ${ }^{24}$ we next investigated the relationship between podoplanin and $\mathrm{E}-$ or N-cadherin in cultured NHEKs and epidermal keratinocytes at the wound edge. Down-regulation of podoplanin by siRNA significantly up-regulated the Ecadherin transcriptional level of NHEKs, whereas no significant change was observed in $\mathrm{N}$-cadherin expression (Figure 3A). Similarly to the results in cultured NHEKs, podoplanin and E-cadherin expression showed inverse correlation in keratinocytes at the wound edge (Figure 3B). At the basal cell layer, keratinocytes showed strong expression of podoplanin and weak expression of E-cadherin, and at the upper layer, they exhibited weak podoplanin and strong E-cadherin expression. In addition, podoplanin and $\mathrm{N}$-cadherin expression appeared to be mostly colocalized (Figure 3B). 

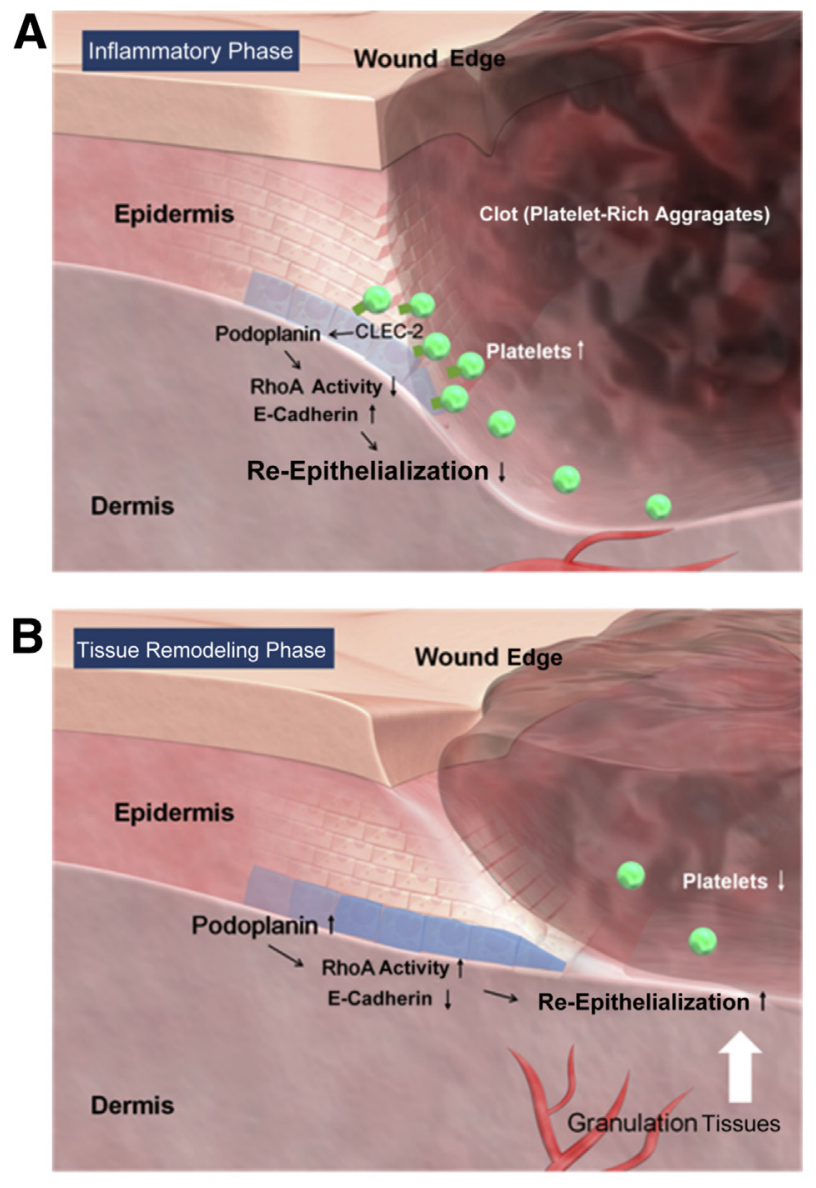

\section{Podoplanin Platelet \& CLEC-2}

Figure 6 Potential functional mechanisms of platelets on wound healing. One of the important functions of platelets on wound healing is to delay re-epithelialization until wound bed preparation is completed. A: During the inflammatory phase, platelets inhibit re-epithelialization on the surface of immature granulation tissue by regulating podoplanin function via C-type lectin-like receptor 2 (CLEC-2). B: During the tissue formation to the remodeling phase, after platelet aggregates are replaced by granulation tissue and bleeding has stopped, the expression of podoplanin is upregulated and re-epithelialization proceeds via RhoA activation and loss of E-cadherin.

Taken together, these results indicate that podoplanin down-regulation resulted in decreased NHEK migration with up-regulation of the cell-cell adhesion molecule, E-cadherin.

\section{Effect of Platelets on NHEK Motility}

Podoplanin is an endogenous ligand for CLEC- $2^{13}$ and induces platelet aggregation by binding to this receptor. ${ }^{13,14}$ Therefore, we sought to determine the role of the podoplanin/CLEC-2 interaction in the effects of platelets on keratinocytes. First, we evaluated the direct adhesion of platelets onto keratinocytes by immunofluorescence staining (Figure 4). One day after wounding, which represents the inflammatory phase of the wound healing process, CD41-positive platelets were observed to be localized at the upper dermis and the basal layer of keratinocytes at the wound edge; podoplanin expression in keratinocytes was not clearly up-regulated. Three days after wounding, which represents the tissue formation phase, the localization of platelets on the upper dermis and the basal cell layer decreased and podoplanin expression in keratinocytes at the wound edge was markedly up-regulated. These findings provided evidence of functional interaction between platelets and keratinocytes. We next investigated the effects of platelets on the motility of NHEKs using the scratch wound assay. After generation of a scratch wound on confluent NHEKs using a pipette tip, $2.0 \times 10^{5}$ platelets $/ \mathrm{mL}$ were added. Platelets significantly inhibited the migration of NHEKs by $40 \%$ (Figure 5A). To evaluate whether the interaction between podoplanin/CLEC-2 contributed to the inhibition of NHEK migration by platelets, 10, 100, or 250 $\mathrm{ng} / \mathrm{mL}$ hCLEC-2 was added to the NHEK culture. The addition of hCLEC-2 inhibited the migration of NHEKs in a dose-dependent manner $(P<0.05)$ (Figure 5B) and up-regulated E-cadherin mRNA (Figure 5C). E-cadherin up-regulation was confirmed by immunofluorescence staining. The E-cadherin signal was strongly positive at the peripheral area of the NHEKs, whereas no significant change was observed in $\beta$-catenin and $\mathrm{N}$-cadherin expression (Figure 5D).

Because RhoA is one of the key mediators of cell migration, RhoA activity was directly measured by G-LISA. RhoA activity was down-regulated by hCLEC- 2 from 1 to 30 minutes after treatment (Figure 5E), suggesting that the regulatory effect of platelets on keratinocyte motility was partly mediated by down-regulating RhoA activity and by losing E-cadherin expression via the podoplanin/CLEC-2 signaling pathway.

The effects of major platelet releasates, such as bone morphogenetic protein-9, PF4, angiostatin, or endostatin, on keratinocyte migration were also examined. However, none of these platelet releasates modulated the migration of NHEKs or podoplanin mRNA levels (data not shown).

Overall, these results indicated that platelets downregulated the function of podoplanin, leading to impaired motility of NHEKs in a CLEC-2-dependent manner.

\section{Discussion}

In this study, the possible functional mechanisms of podoplanin on keratinocytes in wounds were demonstrated. We observed that the expression of podoplanin began to increase shortly after wounding, and reached its peak after several days when bleeding had stopped and the platelet-rich clot was replaced by granulation tissue. Podoplanin up-regulation at the wound edge is thought to be mediated by STAT3b signaling. ${ }^{10}$ Down-regulating podoplanin decreased the motility of keratinocytes, which is essential for reepithelialization during wound healing. Wound healing is a dynamic, interactive process involving soluble mediators, 
blood cells, extracellular matrix, and parenchymal cells. ${ }^{25}$ Wound healing has three phases-inflammation, tissue formation, and tissue remodeling-that overlap in time. Commonly, re-epithelialization is seen from the middle of the tissue formation phase to the end of the tissue remodeling phase, but is not seen in the inflammation phase. Our findings that podoplanin was up-regulated at the time of reepithelialization and that its disruption altered keratinocyte behavior suggested that podoplanin plays a pivotal role in re-epithelialization.

E-cadherin is a transmembrane glycoprotein and a mediator of calcium-dependent cell-cell adhesion in normal cells. ${ }^{26}$ Several studies have reported that reduced expression of E-cadherin correlates with the expression of podoplanin and a poor prognosis in squamous cell carcinoma. ${ }^{9,27,28}$ This suggests that the presence of podoplanin in oral squamous cell carcinoma cells is related to a migratory/ invasive phenotype. ${ }^{9}$ Thus, podoplanin might mediate cell motility via down-regulation of E-cadherin that contributes to the loss of cell adhesion in squamous carcinoma cells. Our study is, perhaps, the first to report that this migratory function of podoplanin is not restricted to tumor cells and is also found in normal keratinocytes during wound healing.

Platelets have multiple roles in wound healing. ${ }^{25}$ During the initial step, tissue injury causes the disruption of blood vessels and extravasation of blood constituents. The blood clot re-establishes hemostasis and provides a provisional extracellular matrix for cell migration. Platelets not only facilitate the formation of a hemostatic plug but also secrete several mediators of wound healing, such as platelet-derived growth factor, that attract and activate macrophages and fibroblasts, which promote granulation tissue formation. ${ }^{25}$ However, the effects of platelets on keratinocytes are not well understood. A single study reported that platelet lysates stimulated the migration and proliferation of keratinocytes. ${ }^{15}$ However, the keratinocytes used therein were immortalized HaCaT cells, in which podoplanin is not expressed at basal levels. ${ }^{15}$ Although we did not evaluate the podoplanin expression in $\mathrm{HaCaT}$ cells under platelet lysate exposure, we suggest that the stimulatory effects of platelets in that study might have been mediated instead by several growth factors released by platelets. On the basis of these findings, we evaluated whether platelets were involved in the migratory function of keratinocytes in wound healing, because platelets contain the CLEC-2 receptor. Our results indicate that platelets down-regulated podoplanin function, which led to a reduction in keratinocyte motility.

According to previous reports ${ }^{25,29}$ and our present findings, we speculate that one of the important functions of platelets on wound healing is to delay re-epithelialization until wound bed preparation is completed (Figure 6). During the inflammatory phase, the wound bed is concave. Reepithelialization within the depressed wound bed results in a severe scar. To prevent this, the platelet-rich aggregates promote the formation of granulation tissue that inhibits re- epithelialization on the surface of immature granulation tissue. During the tissue-remodeling phase, after platelet aggregates are replaced by granulation tissue and bleeding has stopped, the expression of podoplanin is up-regulated and re-epithelialization proceeds.

Finally, we evaluated whether the inhibitory effects of platelets on the motility of keratinocytes was because of direct signaling via CLEC-2 on podoplanin. In accordance with this supposition, we found that the administration of CLEC-2 decreased the motility of keratinocytes. Our results suggest that the concomitant up-regulation of E-cadherin expression and down-regulation of RhoA activity might be possible mechanisms of this inhibitory effect. In addition, a previous report indicated that the podoplanin/CLEC-2 interaction inhibited functions of lymphatic endothelial cells, such as proliferation, migration, and tube formation. ${ }^{30}$ Of these, migration was thought to be inhibited partly through a contact-dependent mechanism because recombinant CLEC-2 inhibited the migration of lymphatic endothelial cells. However, most of these inhibitory effects were because of bone morphogenetic protein- 9 released from platelets that became activated via the podoplanin/CLEC-2 interaction. In accordance with these observations, we investigated which platelet releasate was involved in the inhibitory effects of these cells on keratinocytes. Surprisingly, none of the platelet releasates tested, including bone morphogenetic protein-9, PF4, angiostatin, and endostatin, influenced the effects of podoplanin on keratinocytes, suggesting that the effects and mechanisms of podoplanin/ CLEC-2 signaling in keratinocytes differed from those of platelet releasates.

In conclusion, the current study, although still speculative, provides data showing some possible mechanisms by which platelets might function as an inhibitory mediator on the motility of keratinocytes during wound healing. In addition, we demonstrated that podoplanin plays a pivotal role in re-epithelialization. Thus, the modulation of podoplanin might have therapeutic potential for treating impaired wound healing, such as occurs with diabetic foot ulcers.

\section{Supplemental Data}

Supplemental material for this article can be found at http://dx.doi.org/10.1016/j.ajpath.2015.09.007.

\section{References}

1. Rishi AK, Joyce-Brady M, Fisher J, Dobbs LG, Floros J, VanderSpek J, Brody JS, Williams MC: Cloning, characterization, and development expression of a rat lung alveolar type I cell gene in embryonic endodermal and neural derivatives. Dev Biol 1995, 167: 294-306

2. Breiteneder-Geleff S, Soleiman A, Horvat R, Amann G, Kowalski H, Kerjaschki D: [Podoplanin: a specific marker for lymphatic endothelium expressed in angiosarcoma]. German. Verh Dtsch Ges Pathol 1999, 83:270-275 
3. Kan S, Konishi E, Arita T, Ikemoto C, Takenaka H, Yanagisawa A, Katoh N, Asai J: Podoplanin expression in cancer-associated fibroblasts predicts aggressive behavior in melanoma. J Cutan Pathol 2014, 41:561-567

4. Krishnan H, Ochoa-Alvarez JA, Shen Y, Nevel E, Lakshminarayanan M, Williams MC, Ramirez MI, Miller WT, Goldberg GS: Serines in the intracellular tail of podoplanin (PDPN) regulate cell motility. J Biol Chem 2013, 288:12215-12221

5. Ochoa-Alvarez JA, Krishnan H, Shen Y, Acharya NK, Han M, McNulty DE, Hasegawa H, Hyodo T, Senga T, Geng JG, Kosciuk M, Shin SS, Goydos JS, Temiakov D, Nagele RG, Goldberg GS: Plant lectin can target receptors containing sialic acid, exemplified by podoplanin, to inhibit transformed cell growth and migration. PLoS One 2012, 7:e41845

6. Shen Y, Chen CS, Ichikawa H, Goldberg GS: SRC induces podoplanin expression to promote cell migration. J Biol Chem 2010, 285: 9649-9656

7. Kato Y, Kaneko M, Sata M, Fujita N, Tsuruo T, Osawa M: Enhanced expression of Aggrus (T1alpha/podoplanin), a platelet-aggregationinducing factor in lung squamous cell carcinoma. Tumour Biol 2005, 26:195-200

8. Schacht V, Dadras SS, Johnson LA, Jackson DG, Hong YK, Detmar M: Up-regulation of the lymphatic marker podoplanin, a mucin-type transmembrane glycoprotein, in human squamous cell carcinomas and germ cell tumors. Am J Pathol 2005, 166:913-921

9. Martin-Villar E, Scholl FG, Gamallo C, Yurrita MM, MunozGuerra M, Cruces J, Quintanilla M: Characterization of human PA2.26 antigen (T1alpha-2, podoplanin), a small membrane mucin induced in oral squamous cell carcinomas. Int J Cancer 2005, 113:899-910

10. Honma M, Minami-Hori M, Takahashi H, Iizuka H: Podoplanin expression in wound and hyperproliferative psoriatic epidermis: regulation by TGF-beta and STAT-3 activating cytokines, IFNgamma, IL-6, and IL-22. J Dermatol Sci 2012, 65:134-140

11. Honma M, Fujii M, Iinuma S, Minami-Hori M, Takahashi H, IshidaYamamoto A, Iizuka H: Podoplanin expression is inversely correlated with granular layer/filaggrin formation in psoriatic epidermis. J Dermatol 2013, 40:296-297

12. Suzuki-Inoue K, Fuller GL, Garcia A, Eble JA, Pohlmann S, Inoue O, Gartner TK, Hughan SC, Pearce AC, Laing GD, Theakston RD, Schweighoffer E, Zitzmann N, Morita T, Tybulewicz VL, Ozaki Y, Watson SP: A novel Syk-dependent mechanism of platelet activation by the C-type lectin receptor CLEC-2. Blood 2006, 107:542-549

13. Suzuki-Inoue K, Kato Y, Inoue O, Kaneko MK, Mishima K, Yatomi Y, Yamazaki Y, Narimatsu H, Ozaki Y: Involvement of the snake toxin receptor CLEC-2, in podoplanin-mediated platelet activation, by cancer cells. J Biol Chem 2007, 282:25993-26001

14. Kato Y, Kaneko MK, Kunita A, Ito H, Kameyama A, Ogasawara S, Matsuura N, Hasegawa Y, Suzuki-Inoue K, Inoue O, Ozaki Y, Narimatsu H: Molecular analysis of the pathophysiological binding of the platelet aggregation-inducing factor podoplanin to the C-type lectin-like receptor CLEC-2. Cancer Sci 2008, 99:54-61

15. Ranzato E, Patrone M, Mazzucco L, Burlando B: Platelet lysate stimulates wound repair of HaCaT keratinocytes. Br J Dermatol 2008, 159:537-545

16. Martin-Villar E, Megias D, Castel S, Yurrita MM, Vilaro S, Quintanilla M: Podoplanin binds ERM proteins to activate RhoA and promote epithelial-mesenchymal transition. J Cell Sci 2006, 119: $4541-4553$

17. Greenhalgh DG, Sprugel KH, Murray MJ, Ross R: PDGF and FGF stimulate wound healing in the genetically diabetic mouse. Am J Pathol 1990, 136:1235-1246

18. Asai J, Takenaka H, Hirakawa S, Sakabe J, Hagura A, Kishimoto S, Maruyama K, Kajiya K, Kinoshita S, Tokura Y, Katoh N: Topical simvastatin accelerates wound healing in diabetes by enhancing angiogenesis and lymphangiogenesis. Am J Pathol 2012, 181: $2217-2224$

19. Asai J, Takenaka H, Katoh N, Kishimoto S: Dibutyryl cAMP influences endothelial progenitor cell recruitment during wound neovascularization. J Invest Dermatol 2006, 126:1159-1167

20. Asai J, Takenaka H, Kusano KF, Ii M, Luedemann C, Curry C, Eaton E, Iwakura A, Tsutsumi Y, Hamada H, Kishimoto S, Thorne T, Kishore R, Losordo DW: Topical sonic hedgehog gene therapy accelerates wound healing in diabetes by enhancing endothelial progenitor cell-mediated microvascular remodeling. Circulation 2006, $113: 2413-2424$

21. Committee for the Update of the Guide for the Care and Use of Laboratory Animals; National Research Council: Guide for the Care and Use of Laboratory Animals: Eighth Edition. Washington, DC, National Academies Press, 2011

22. Suzuki-Inoue K, Inoue O, Frampton J, Watson SP: Murine GPVI stimulates weak integrin activation in PLCgamma2-/- platelets: involvement of PLCgamma1 and PI3-kinase. Blood 2003, 102:1367-1373

23. Abràmoff MD, Magalhães PJ, Ram SJ: Image processing with ImageJ. Biophoto Int 2004, 11:36-42

24. Cavallaro U, Schaffhauser B, Christofori G: Cadherins and the tumour progression: is it all in a switch? Cancer Lett 2002, 176:123-128

25. Singer AJ, Clark RA: Cutaneous wound healing. N Engl J Med 1999, 341:738-746

26. Wijnhoven BP, Dinjens WN, Pignatelli M: E-cadherin-catenin cell-cell adhesion complex and human cancer. Br J Surg 2000, 87:992-1005

27. Nakashima Y, Yoshinaga K, Kitao H, Ando K, Kimura Y, Saeki H, Oki E, Morita M, Kakeji Y, Hirahashi M, Oda Y, Maehara Y: Podoplanin is expressed at the invasive front of esophageal squamous cell carcinomas and is involved in collective cell invasion. Cancer Sci 2013, 104:1718-1725

28. Toll A, Masferrer E, Hernandez-Ruiz ME, Ferrandiz-Pulido C, Yebenes M, Jaka A, Tuneu A, Jucgla A, Gimeno J, Baro T, Casado B, Gandarillas A, Costa I, Mojal S, Pena R, de Herreros AG, Garcia-Patos V, Pujol RM, Hernandez-Munoz I: Epithelial to mesenchymal transition markers are associated with an increased metastatic risk in primary cutaneous squamous cell carcinomas but are attenuated in lymph node metastases. J Dermatol Sci 2013, 72: 93-102

29. Galliera E, Corsi MM, Banfi G: Platelet rich plasma therapy: inflammatory molecules involved in tissue healing. J Biol Regul Homeost Agents 2012, 26:35S-42S

30. Osada $\mathrm{M}$, Inoue $\mathrm{O}$, Ding $\mathrm{G}$, Shirai $\mathrm{T}$, Ichise $\mathrm{H}$, Hirayama $\mathrm{K}$, Takano K, Yatomi Y, Hirashima M, Fujii H, Suzuki-Inoue K, Ozaki Y: Platelet activation receptor CLEC-2 regulates blood/ lymphatic vessel separation by inhibiting proliferation, migration, and tube formation of lymphatic endothelial cells. J Biol Chem 2012, 287: 22241-22252 